Check for updates

Cite this: Chem. Sci., 2019, 10, 5906

๑ All publication charges for this article have been paid for by the Royal Society of Chemistry

\section{Quo vadis niobium? Divergent coordination behavior of early-transition metals towards MOF- $5 \dagger$}

\author{
Maciej D. Korzyński, (D) a Luca Braglia, (DD ${ }^{\mathrm{b}}$ Elisa Borfecchia, (D) cd \\ Kirill A. Lomachenko, (D) Amgalanbaatar Baldansuren, (D) ${ }^{f}$ \\ Christopher H. Hendon, (D) ${ }^{9}$ Carlo Lamberti (D) thi and Mircea Dincă (D) *a
}

Received 29th March 2019

Accepted 7th May 2019

DOI: $10.1039 / c 9 s c 01553 a$

rsc.li/chemical-science
Treatment of MOF-5 with $\mathrm{NbCl}_{4}(\mathrm{THF})_{2}$ in acetonitrile leads to incorporation of $\mathrm{Nb}$ (IV) centers in a fashion that diverges from the established cation metathesis reactivity of this iconic material. A combination of $\mathrm{X}$-ray absorption spectroscopy analysis and reactivity studies altogether supported by density functional theory computational studies document an unprecedented binding mode for the $\mathrm{Zn}_{4} \mathrm{O}\left(\mathrm{O}_{2} \mathrm{C}-\right)_{6}$ secondary building units (SBUs), which in Nb(IV)-MOF-5 function as $\kappa^{2}$-chelating ligands for $\mathrm{NbCl}_{4}$ moieties, with no exchange of $\mathrm{Zn}^{2+}$ observed. This unusual reactivity expands the portfolio of postsynthetic modification techniques available for MOFs, exemplified here by MOF-5, and underscores the diverse coordination environments offered by this and potentially other MOFs towards heterometal species.

\section{Introduction}

Nominally, the compositional variety of metal-organic frameworks (MOFs) is unlimited, with virtually any stable metal in the periodic table potentially serving as the basis for the inorganic building blocks, or the secondary building units (SBUs). ${ }^{1-9}$ Yet, the vast majority of SBUs are made from a very limited set of metal species, nearly all MOFs featuring first-row transition metals, with heavy representation among the late first row metals. Although early transition metal (ETM)-based MOFs have distinguished themselves with remarkable stability, especially

\footnotetext{
${ }^{a}$ Department of Chemistry, Massachusetts Institute of Technology, 77 Massachusetts Avenue, Cambridge, MA 02139, USA. E-mail: mdinca@mit.edu

${ }^{b}$ CNR-Istituto Officina dei Materiali, TASC Laboratory in Area Science Park - Basovizza, Strada Statale $14 \mathrm{~km}$ 163.5, 34149 Trieste, Italy

'Department of Chemistry, NIS, CrisDi, INSTM Centre of Reference, University of Turin, Via Quarello 15, I-10135 Torino, Italy

${ }^{d}$ Center for Materials Science and Nanotechnology (SMN), Department of Chemistry, University of Oslo, 1033 Blindern, 0315 Oslo, Norway

${ }^{e}$ European Synchrotron Radiation Facility, 71 Avenue des Martyrs, CS 40220, 38043 Grenoble Cedex 9, France

${ }^{f}$ EPSRC National EPR Facility, School of Chemistry, The University of Manchester, Oxford Road, Manchester M13 9PL, UK

${ }^{g}$ Materials Science Institute, Department of Chemistry and Biochemistry, University of Oregon, Eugene, Oregon 97403, USA

${ }^{h}$ Department of Physics, NIS, CrisDi, Interdepartmental Centers, INSTM Centre of Reference, University of Turin, Via Giuria 1, I-10125 Torino, Italy

${ }^{i}$ The Smart Materials Research Institute, Southern Federal University, 178/24 Sladkova Street, Rostov-on-Don, 344090, Russia

$\dagger$ Electronic supplementary information (ESI) available. See DOI: 10.1039/c9sc01553a

‡ Deceased February 1, 2019.
}

under aqueous conditions, ${ }^{10}$ MOFs made from ETMs are still rare outside of $\mathrm{Zr}$ and Ti. One of the major challenges associated with de novo synthesis of ETM-based MOFs is the tendency of metal precursors to undergo deleterious oxygen-scavenging reactivity under solvothermal conditions encountered in typical MOF syntheses. ${ }^{11}$

With an eye towards enriching the compositional space of MOFs with other ETMs and to explore the fundamentals of bonding for ETMs within this set of materials, we turned our attention to niobium, which remains essentially unexplored in this sense. Indeed, to our knowledge, there are only two reported Nb-containing frameworks in the literature. ${ }^{12,13}$ With its flexible coordination sphere, large covalent radius, and extreme oxophilicity, niobium is the epitome of a difficult substrate for MOF formation and should therefore provide insight into the behavior of other early transition metals for this purpose. ${ }^{14}$

To circumvent the expected challenges associated with the direct synthesis of Nb MOFs (vide supra), we decided to focus on post-synthetic modification (PSM), a method that has previously proven successful in altering the chemical structure of pre-formed MOFs. ${ }^{15-17}$ Because PSM allows for mild reaction conditions, contrasting with the harsh solvothermal synthetic routes encountered in de novo MOF preparation, it can lead to the isolation of materials that are difficult to form otherwise. ${ }^{18}$ In particular, within the context of post-synthetic cation exchange ${ }^{19}$ our group and others have shown, initially through the iconic $\mathrm{Zn}_{4} \mathrm{O}(\mathrm{BDC})_{3}$ (MOF-5; BDC = terephthalate) (Fig. 1a), that the SBUs within MOFs can be thought of as supramolecular chelating ligands for incoming metal ions. The exchanged SBUs behave spectroscopically and chemically as isolated molecules with unusual coordination environments that are often 
a)

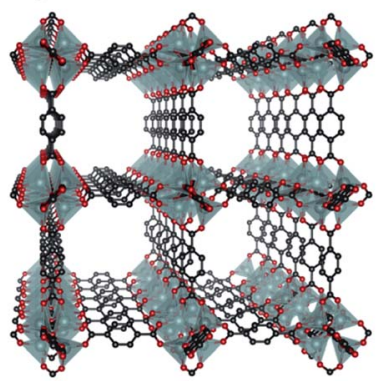

b)

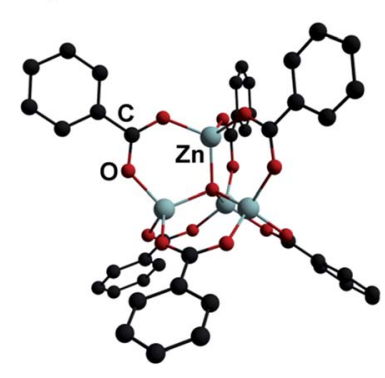

c)
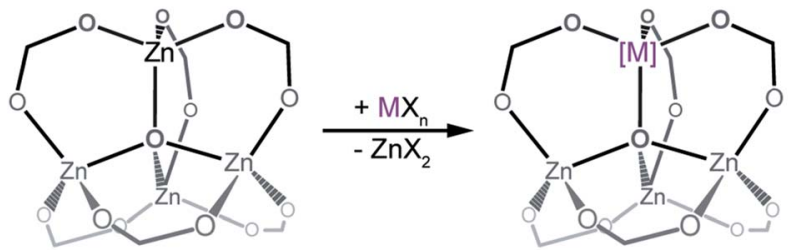

$[\mathrm{M}]=\mathrm{MX}_{\mathrm{n}-2}$

Fig. 1 (a) Structure of MOF-5, (b) its SBU, and (c) idealized cation exchange process. Hydrogen atoms were omitted for clarity.

unknown from traditional solution chemistry. ${ }^{20}$ These earlier studies showed that the $\left[\mathrm{Zn}_{4} \mathrm{O}\right]^{6+}$ SBU (Fig. 1b) is particularly prone to exchanging $\mathrm{Zn}^{2+}$ for the majority of the first row transition metal cations in various oxidation states (Fig. 1c). ${ }^{21-23}$ MOF was therefore identified as a promising platform for exploring $\mathrm{Nb}$ chemistry. Herein, we document our quest in understanding niobium immobilization in MOF-5, and we unveil an unprecedented coordination mode within its SBU.

\section{Results and discussion}

Appropriate starting materials are available for $\mathrm{Nb}$ in several oxidation states, with the $\mathrm{Nb}$ (Iv) $\mathrm{d}^{1}$ system offering an additional characterization handle in the form of electron paramagnetic resonance (EPR) spectroscopy, when compared to the more common $\mathrm{Nb}(\mathrm{v})$ oxidation state. To this end, the soluble
niobium(Iv) chloride tetrahydrofuran complex $\left(\mathrm{NbCl}_{4}(\mathrm{THF})_{2}\right)$ serves as a good entry point to $\mathrm{Nb}$ (Iv) chemistry. ${ }^{24}$ The routine method to introduce a foreign metal into a framework involves soaking the MOF crystals in a solution of the desired precursor. Here, treatment of pre-activated, colorless MOF-5 crystals ${ }^{25}$ with a yellow-green solution of $\mathrm{NbCl}_{4}(\mathrm{THF})_{2}$ in acetonitrile (MeCN) led to an almost instantaneous change of color for the crystals to dark violet (Fig. S1†). The observed fast kinetics of this process were surprising given that reported first row cation exchange processes proceed rather slowly. After $48 \mathrm{~h}$, the isolated dark violet crystals of $\mathrm{Nb}$ (Iv)-treated MOF-5 were washed extensively with MeCN then dichloromethane (DCM) until no characteristic features of $\mathrm{Nb}$ (Iv) could be observed in the UV spectrum of the effluent solutions. Residual solvent molecules within the pores and/or weakly-bound to the metal centers, were removed by heating to $150{ }^{\circ} \mathrm{C}$ under high vacuum (0.1 mTorr).

Powder X-ray diffraction (PXRD) analysis confirmed that treatment of MOF-5 with the niobium solution and subsequent thermal treatment proceed with preservation of crystallinity (Fig. S2 $\dagger)$. The $R_{1}\left(9.7^{\circ}\right.$-to-6. $\left.8^{\circ}\right)$ and $R_{2}\left(13.8^{\circ}\right.$-to- $\left.6.8^{\circ}\right)$ peak ratios $^{\mathbf{2 6 , 2 7}}$ were low indicating no pore obstruction by the guest molecules and lack of framework interpenetration. Inspection of the attenuated total reflection Fourier-transform infrared (ATR-FTIR) spectrum of the activated material showed predominantly typical MOF-5 features (Fig. S3†). Additional FTIR bands in the spectrum of $\mathrm{Nb}$ (Iv)-treated MOF-5, which are absent in the spectrum of MOF-5 itself, are also present in the vacuum-dried residue obtained from a solution of $\mathrm{NbCl}_{4}(\mathrm{THF})_{2}$ in acetonitrile (Fig. S4 $\dagger$ ). In particular, a prominent feature at $2282 \mathrm{~cm}^{-1}$ in this latter residue suggests that excess acetonitrile partially replaces THF in the primary coordination sphere of $\mathrm{Nb}$ (Iv), in agreement with the literature data on MeCN adducts of $\mathrm{Nb}(\mathrm{Iv}),{ }^{28}$ and is likely carried over upon reaction with MOF-5. Importantly, this band does not persist in fully activated $\mathrm{Nb}$ (Iv)MOF-5, indicating full removal of MeCN from this material.

The documented tendency of $\mathrm{Nb}(\mathrm{Iv})$ compounds to disproportionate thermally ${ }^{29}$ required confirmation that niobium immobilization within MOF-5 occurs with preservation of the +IV oxidation state, which came from EPR spectroscopy and X-ray a)

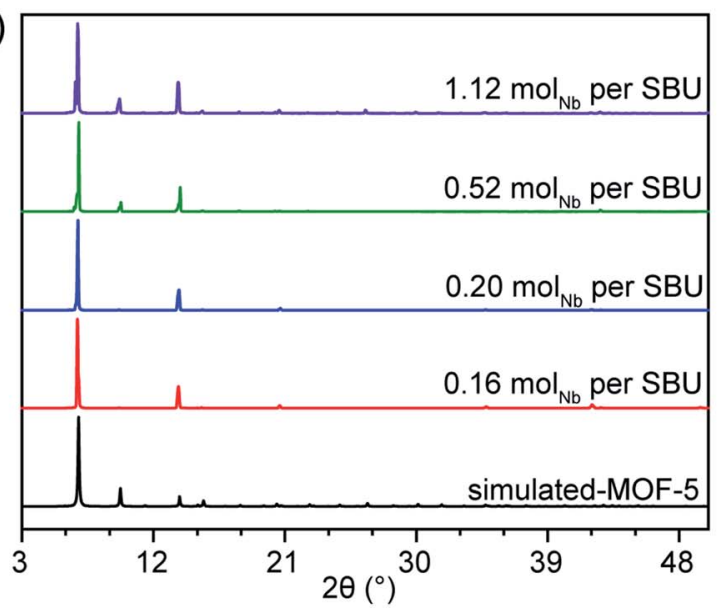

b)

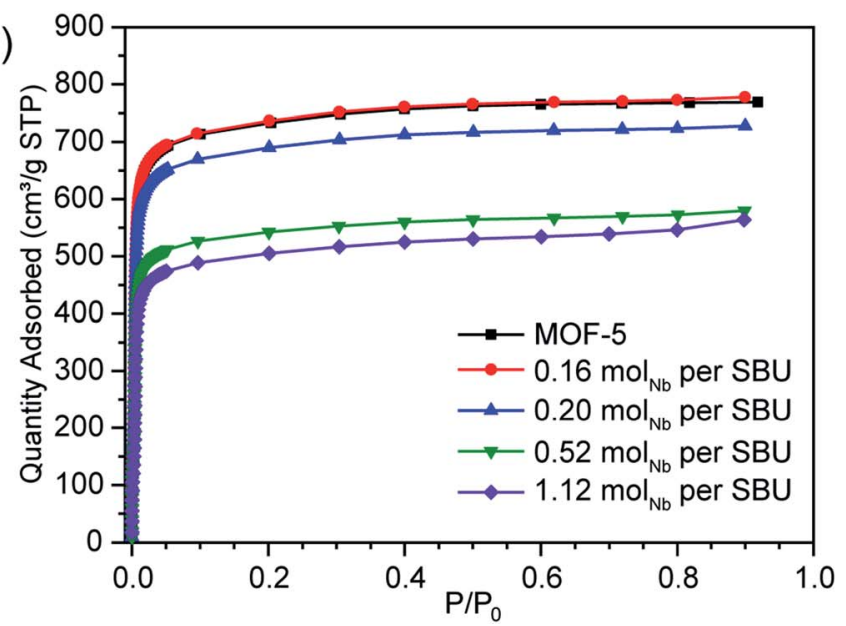

Fig. 2 (a) PXRD patterns and (b) $\mathrm{N}_{2}$ adsorption isotherms for $\mathrm{Nb}$ (IV)-treated MOF-5 samples with increasing niobium content. 
absorption spectroscopy (XAS). Although the typical hyperfine splitting pattern for ${ }^{93} \mathrm{Nb}(\mathrm{I}=9 / 2)$ is observed in the EPR spectrum of $\mathrm{Nb}$ (Iv)-MOF-5 at X-band, the peaks are significantly broadened, with a first-order hyperfine splitting $\left(A_{\mathrm{Nb}}\right)$ of approximately $557 \mathrm{MHz}$ (Fig. 3, Table $\mathrm{S} 1 \dagger$ ). In the simulated spectrum (Fig. S5 and S6 $\dagger$ ), the hyperfine interactions of both isotopes ${ }^{35} \mathrm{Cl}$ and ${ }^{37} \mathrm{Cl}$ (both $\mathrm{I}=3 / 2$ nuclei) are included, and although they do not affect the alignment of the electron spins, these contribute to inhomogeneous line broadening. Notably, EPR signals obtained for $\mathrm{Nb}(\mathrm{Iv})-\mathrm{MOF}-5$ and a toluene-MeCN frozen glass of $\mathrm{NbCl}_{4}(-$ $\mathrm{THF})_{2}$ are similar, the aforementioned line broadening in the MOF notwithstanding (Fig. S7†). Finally, analysis of the XAS data in the $\mathrm{Nb} \mathrm{K}$-edge $\mathrm{X}$-ray absorption near edge structure region (XANES) for $\mathrm{Nb}$ (Iv)-treated MOF-5 and a series of $\mathrm{Nb}(\mathrm{Iv})$ standards $^{30}$ confirmed that the edge energy, $\approx 19001 \mathrm{eV}$, was in line with that of niobium(Iv) oxide (Fig. S8†). These results offer confidence that $\mathrm{Nb}(\mathrm{Iv})$ does not disproportionate and remains in its original oxidation state when immobilized within MOF-5.

The impact of immobilized $\mathrm{Nb}$ content on the properties of the MOF was probed by preparing samples containing different Nb-to-SBU molar ratios $(0.16,0.20,0.52$, and 1.12 ; determined by inductively coupled plasma - atomic emission spectroscopy, ICP-AES). Even when using less than one equivalent of $\mathrm{Nb}$ precursor with respect to the $\left[\mathrm{Zn}_{4} \mathrm{O}\right]^{6+} \mathrm{SBU}, \mathrm{Nb}$ uptake into the MOF progressed rapidly and in a qualitatively similar manner, with retention of crystallinity (Fig. 2a). This stands in contrast with cation exchange, which occurs typically only in an excess of incoming metal species and often leads to loss of crystallinity when exchange is rapid.

The porosity of the $\mathrm{Nb}(\mathrm{Iv})$-treated samples was further confirmed by $\mathrm{N}_{2}$ adsorption isotherms at $77 \mathrm{~K}$ (Fig. 2b). Fitting these to the Brunauer-Emmett-Teller (BET) equation gave apparent surface areas ranging from $2943 \mathrm{~m}^{2} \mathrm{~g}^{-1}$ to $2010 \mathrm{~m}^{2} \mathrm{~g}^{-1}$ for $\mathrm{Nb}$ (Iv)-MOF-5 samples with $0.16 \mathrm{~mol} \mathrm{Nb}$ per SBU and $1.12 \mathrm{~mol}$ $\mathrm{Nb}$ per SBU, respectively. Although these are lower than anticipated for cation-exchanged materials, the decreased porosity was not caused by surface deposition of certain species that would

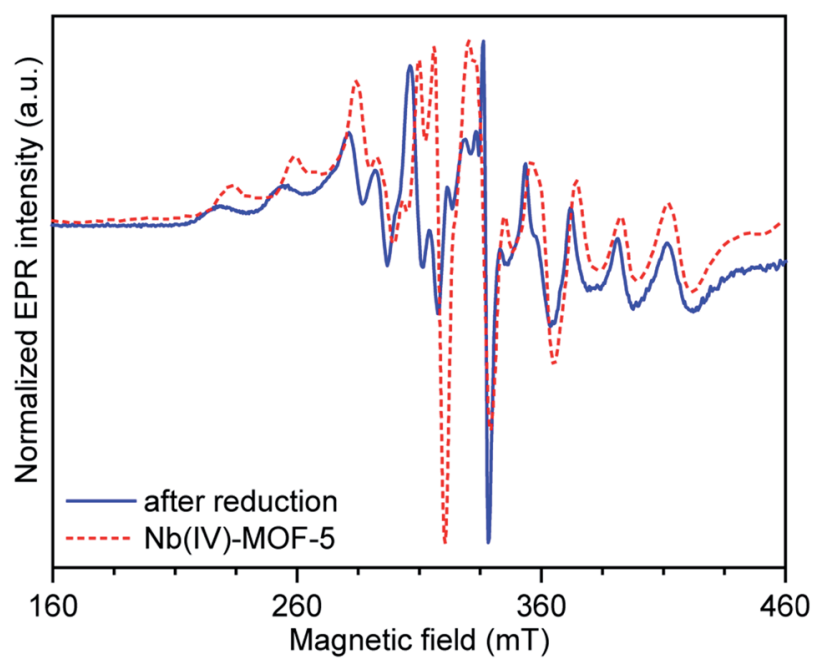

Fig. 3 EPR spectra of $\mathrm{Nb}(\mathrm{IV})-\mathrm{MOF}-5$ and of $\mathrm{Nb}(\mathrm{v})-\mathrm{MOF}-5$ treated with $(\mathrm{TMS})_{2}$ pyr. block access to the pores. Indeed, scanning electron microscopy energy dispersive X-ray spectroscopy mapping revealed that niobium deposition occurs not only on the surface, but also homogeneously throughout the crystal (Fig. S9 and S10†).

With solid evidence that $\mathrm{Nb}$ is immobilized inside the pores of MOF-5, but with conflicting evidence regarding its substitution of $\mathrm{Zn}^{2+}$ ions inside the SBU, the primary coordination environment of $\mathrm{Nb}$ (Iv) within MOF- 5 was probed by XAS. ${ }^{\mathbf{3 1}}$ These studies were aided by density functional theory (DFT) calculations that modelled three $\mathrm{Nb}$ (Iv) bonding scenarios. The first model assumed that typical cation exchange occurred, with a $\left[\mathrm{NbCl}_{2}\right]^{2+}$ unit replacing a $\mathrm{Zn}^{2+}$ ion to produce a neutral $\mathrm{Cl}_{2}$ $\mathrm{NbZn}_{3} \mathrm{O}\left(\mathrm{O}_{2} \mathrm{C}-\right)_{6}$ cluster (Fig. 4a). As shown with $\mathrm{Ni}^{2+}$-exchanged MOF- $5,{ }^{21}$ the SBU in this material is able to support octahedral metal ions, as would be required for the $\mathrm{Nb}$ center in this model. A second model assumed that the $\left[\mathrm{Zn}_{4} \mathrm{O}\right]^{6+}$ constitution of the node is preserved and that instead of exchanging any $\mathrm{Zn}^{2+}$ ions, a $\mathrm{NbCl}_{4}$ moiety binds to the SBU in an exogenous $\kappa^{2}$ fashion utilizing two carboxylate oxygen atoms as anchor points (Fig. 4b). Finally, to account for the possibility of adventitious oxygen or water scavenging during the deposition, we also considered a niobyl unit $\left(\mathrm{NbOCl}_{2}\right)$ bound to the SBU exogenously in a $\kappa^{2}$-fashion similar to the $\mathrm{NbCl}_{4}$ model (Fig. $4 \mathrm{c}$ ).

The XAS data coupled with these DFT models was sufficient to discriminate the local $\mathrm{Nb}$ environment within MOF-5. Extended X-ray absorption fine structure (EXAFS) analysis of the XAS data for a wide range of $\mathrm{Nb}^{30}$ allowed adoption of a twostep refinement strategy to analyze the experimental EXAFS spectrum of $\mathrm{Nb}(\mathrm{Iv})-\mathrm{MOF}-5$ : (i) in a first step, all the parameters for the three DFT-structures were optimized (Table S2 $\dagger$ ) apart from the $S_{0}{ }^{2}$, which was fixed to 1 and the Debye-Waller factor of $\mathrm{O}, \sigma_{\mathrm{O}}{ }^{2}$, and of $\mathrm{Cl},{\sigma_{\mathrm{Cl}}}^{2}$, which were fixed from the EXAFS results obtained on the reference samples; (ii) the second step involved refinement of $S_{0}{ }^{2}$ (Table S3 $\dagger$ ). Unexpectedly, the first step revealed that the fit for ' $\mathrm{NbCl}_{4}$ ' matched the experimental data much better than other models. Confirming that no hydrolysis at the $\mathrm{Nb}$ center occurs, the fit for the ' $\mathrm{NbOCl}_{2}$ ' model yielded a meaningless value of $\Delta E(15 \pm 31) \mathrm{eV}$ and an unphysically high $\sigma^{2}(0.14 \pm 0.12) \AA^{2}$ for the scattering paths involving $\mathrm{C}$ and $\mathrm{O}$ atoms at distances larger than $3.2 \AA$ from the $\mathrm{Nb}$ absorber. Although the parameters obtained for the ' $\mathrm{NbCl}_{2}$ ', cation exchange model were also reasonable, the fit for this model did not match the experimental curves as well as the ' $\mathrm{NbCl}_{4}$ ' fit. Indeed, the $R$-factor for the ' $\mathrm{NbCl}_{2}$ ' model was seven times larger than that for ' $\mathrm{NbCl}_{4}$ ' (Table $\mathrm{S} 2 \dagger$ ).
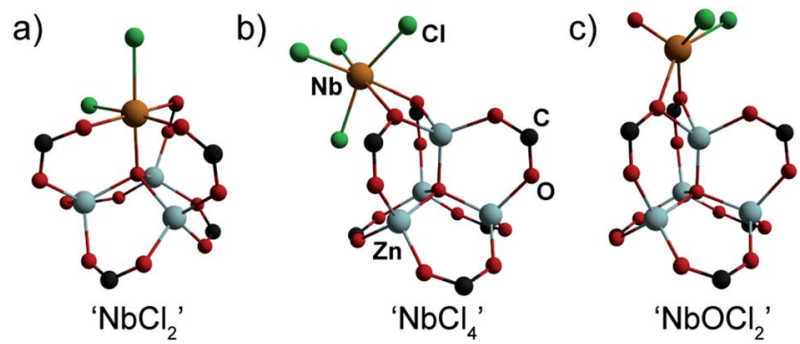

Fig. 4 DFT-optimized models of plausible $\mathrm{Nb}$ coordination modes in MOF-5. 
The second step in the EXAFS fitting routine (Table S3 $\dagger$ ) definitely disqualified the cation exchanged (' $\left.\mathrm{NbCl}_{2}{ }^{\prime}\right)$ model as a likely candidate for $\mathrm{Nb}(\mathrm{Iv})$-MOF-5. Indeed, optimizing the $S_{0}{ }^{2}$ for all three models excluded ' $\mathrm{NbCl}_{2}$ ' both on the basis of an unreasonably high value for the energy shift $(\Delta E=10 \pm 2 \mathrm{eV})$ and a value that was too low for the $S_{0}{ }^{2}$ parameter $(0.51 \pm 0.04)$. Lastly, the best qualitative agreement between the fit and experimental curves was obtained for the ' $\mathrm{NbCl}_{4}$ ' model (Fig. 5, red curves), which we therefore favor as the dominant coordination environment of $\mathrm{Nb}$ in $\mathrm{Nb}(\mathrm{rv})-\mathrm{MOF}-5$. The final fit parameters for this model (Fig. S11 $\dagger$ ) gave $\mathrm{Nb}-\mathrm{O}$ distances of 2.49(5) $\AA$ and two independent sets of $\mathrm{Cl}$ atoms situated at 2.23(4) $\AA$ and 2.41(2) $\AA$ from the niobium center.

Immobilization of hetero-metal species in MOF- 5 by means other than cation exchange has received little attention in the literature. In fact, the vast majority of examples that employ decoration of the SBUs with exogenous metals, rather than cation exchange, are reported for Zr-based materials. ${ }^{16,32-35}$ One recent example with transition metals in MOF-5 involved the installation of $\mathrm{Fe}(\mathrm{OH})_{3}\left(\mathrm{H}_{2} \mathrm{O}\right)_{2}$ moieties bridging SBUs on two interpenetrated lattices, ${ }^{36}$ with limited additional examples focusing on alkali metal decoration of MOF-5. ${ }^{37-42}$ These findings point to a previously unrecognized divergence in the reactivity of early and late transition metals with oxygen-based SBUs: whereas the late transition metals favor cation exchange reactivity (the Zr-based MOFs notwithstanding ${ }^{43}$ ), we show here that the high-valent early transition metals prefer to adhere to the SBU rather than exchange within the SBU, presumably because of their extreme oxophilicity. Notably, MOF-5 is a rare instance that allows both processes to occur.§ Further proof of exogenous SBU binding of $\mathrm{Nb}(\mathrm{rv})$, rather than exchange within the SBU, came from a clean reaction between $\mathrm{Nb}(\mathrm{rv})-\mathrm{MOF}-5$ and $\mathrm{XeF}_{2}$ in $\mathrm{MeCN}$, which caused a swift color change of the violet crystals to off-white and emergence of a yellow solution. Inspection of the off-white solid by ICP-AES showed that niobium was almost quantitatively removed from the material, with preservation of crystallinity (Fig. S12†). This would be unlikely if niobium served a structural role as part of the SBU.

Immobilization of early transition metal ions by decoration of the MOF- $5 \mathrm{SBU}$ can be extended to $\mathrm{Nb}(\mathrm{v})$, which furthermore
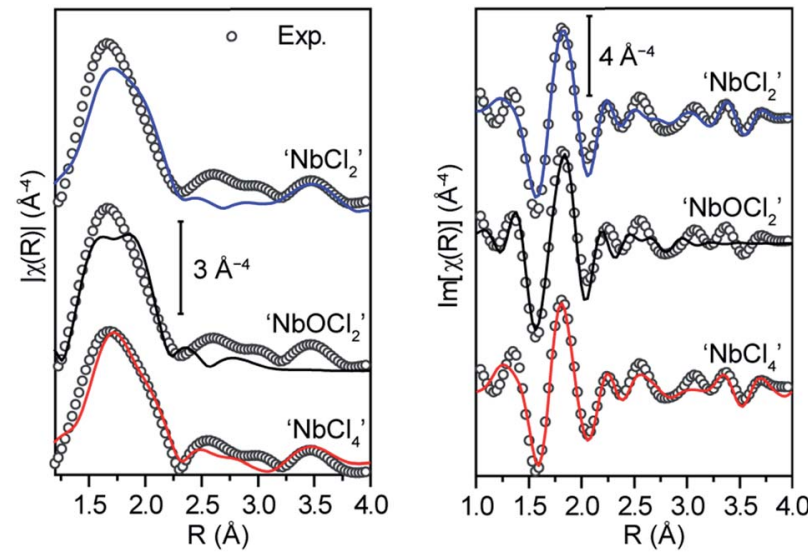

Fig. 5 Comparison of EXAFS fits for various models of $\mathrm{Nb}$ moieties within MOF-5. engages in molecule-like, well behaved redox chemistry within the MOF, reminiscent of the behavior of late transition metals in the same material. Thus, soaking MOF-5 in a solution of $\mathrm{NbCl}_{5}$ in MeCN yielded $\mathrm{Nb}(\mathrm{v})$-MOF-5 as an off-white crystalline powder. Despite the higher Lewis acidity ${ }^{44}$ and even higher oxophilicity of $\mathrm{Nb}(\mathrm{v})$ relative to $\mathrm{Nb}(\mathrm{Iv}), \mathrm{Nb}(\mathrm{v})$-decorated MOF-5 also retains crystallinity (Fig. S14†) and, upon activation in a similar manner to the $\mathrm{Nb}$ (Iv) MOF, shows porosity with a BET surface area of $1437 \mathrm{~m}^{2} \mathrm{~g}^{-1}$ (Fig. S16†). This value is lower than that of $\mathrm{Nb}$ (Iv)-MOF-5, as may be expected for a material with an additional chloride protruding into the pore. A testament to the molecule-like, site-isolated environment of $\mathrm{Nb}(\mathrm{v})$ within this material was its clean reduction with 2,3,5,6-tetramethyl-1,4bis(trimethylsilyl)-1,4-diaza-2,5-cyclohexadiene ((TMS $)_{2}$ pyr), a practical reagent for the reduction of high-valent early transition metal halides. ${ }^{45}$ Treatment of $\mathrm{Nb}(\mathrm{v})$-MOF- 5 with an excess of (TMS $)_{2}$ pyr in THF led to rapid change of color of the solids from off-white to dark blue, a transformation that once again occurred with preservation of crystallinity (Fig. S17†). The reduced MOF exhibited an X-band EPR spectrum in THF that was qualitatively similar to that of independently-synthesized $\mathrm{Nb}$ (Iv)-MOF-5 obtained from $\mathrm{NbCl}_{4}(\mathrm{THF})_{2}$ (Fig. 3, blue trace). Although a simple inner-sphere electron transfer reaction, this transformation demonstrates that metal centers decorating the MOF-5 SBU are amenable for further synthetic elaboration in a manner similar to those exchanged into the SBU.

\section{Conclusions}

In our endeavors to advance the niobium chemistry in MOFs, we discovered a novel coordination mode of foreign metal species to the SBU of MOF-5. Our results show that it is possible both to introduce and to remove niobium ions with the preservation of the parent framework's crystallinity. The chemical competence of these extra framework $\mathrm{Nb}$ sites is exemplified by simple reduction of $\mathrm{Nb}(\mathrm{v})$ to $\mathrm{Nb}(\mathrm{Iv})$ within the framework. Excitingly, literally 20 years after its initial discovery, MOF-5, arguably one of the most iconic materials in its class, continues to surprise. This bodes well for the richness of fundamental chemistry yet to be discovered with the other thousands of MOFs out there.

\section{Conflicts of interest}

There are no conflicts to declare.

\section{Acknowledgements}

This work was supported through a CAREER grant to M. D. from the National Science Foundation (DMR-1452612). Computational work used the Extreme Science and Engineering Discovery Environment (XSEDE), which is supported by National Science Foundation grant number ACI-1548562. C. L. acknowledges the Mega-grant of the Russian Federation Government, No. 14.Y26.31.0001. The authors would like to acknowledge Dr Wesley J. Transue for aid with computational work, Dr Lei Sun for assistance in SEM-EDX measurements, Dr 
Giovanni Agostini for the assistance during the experiment at BM23 beamline ${ }^{46}$ of the ESRF and Prof. Dr Nicholas Chilton for helpful discussions regarding the EPR data.

\section{Notes and references}

$\S$ Even though it has been reported that SBUs undergo cation exchange in the UiO66 framework, it was later shown that deposition of the foreign metal oxide was instead occurring, see ref. 43.

1 J.-R. Li, J. Sculley and H.-C. Zhou, Chem. Rev., 2012, 112, 869932.

2 H. Li, K. Wang, Y. Sun, C. T. Lollar, J. Li and H.-C. Zhou, Mater. Today, 2018, 21, 108-121.

3 L. E. Kreno, K. Leong, O. K. Farha, M. Allendorf, R. P. Van Duyne and J. T. Hupp, Chem. Rev., 2012, 112, 1105-1125.

4 F.-Y. Yi, D. Chen, M.-K. Wu, L. Han and H.-L. Jiang, Chempluschem, 2016, 81, 675-690.

5 W. P. Lustig, S. Mukherjee, N. D. Rudd, A. V. Desai, J. Li and S. K. Ghosh, Chem. Soc. Rev., 2017, 46, 3242-3285.

6 D. Farrusseng, S. Aguado and C. Pinel, Angew. Chem., Int. Ed., 2009, 48, 7502-7513.

7 C. A. Downes and S. C. Marinescu, ChemSusChem, 2017, 10, 4374-4392.

8 N. S. Bobbitt, M. L. Mendonca, A. J. Howarth, T. Islamoglu, J. T. Hupp, O. K. Farha and R. Q. Snurr, Chem. Soc. Rev., 2017, 46, 3357-3385.

9 J.-S. Qin, S. Yuan, C. Lollar, J. Pang, A. Alsalme and H.-C. Zhou, Chem. Commun., 2018, 54, 4231-4249.

10 S. Yuan, L. Feng, K. Wang, J. Pang, M. Bosch, C. Lollar, Y. Sun, J. Qin, X. Yang, P. Zhang, Q. Wang, L. Zou, Y. Zhang, L. Zhang, Y. Fang, J. Li and H.-C. Zhou, Adv. Mater., 2018, 30, 1704303.

11 T. Devic and C. Serre, Chem. Soc. Rev., 2014, 43, 6097-6115.

12 A. Cadiau, K. Adil, P. M. Bhatt, Y. Belmabkhout and M. Eddaoudi, Science, 2016, 353, 137-140.

13 S. Ahn, N. E. Thornburg, Z. Li, T. C. Wang, L. C. Gallington, K. W. Chapman, J. M. Notestein, J. T. Hupp and O. K. Farha, Inorg. Chem., 2016, 55, 11954-11961.

14 L. G. Hubert-Pfalzgraf, Niobium \& Tantalum: Inorganic \& Coordination Chemistry in Encyclopedia of Inorganic Chemistry, ed. R. A. Scott, John Wiley \& Sons, Ltd, 2006.

15 K. K. Tanabe and S. M. Cohen, Chem. Soc. Rev., 2011, 40, 498-519.

16 R. J. Marshall and R. S. Forgan, Eur. J. Inorg. Chem., 2016, 2016, 4310-4331.

17 Z. Yin, S. Wan, J. Yang, M. Kurmoo and M.-H. Zeng, Coord. Chem. Rev., 2019, 378, 500-512.

18 N. Stock and S. Biswas, Chem. Rev., 2012, 112, 933-969.

19 C. K. Brozek and M. Dincă, Chem. Soc. Rev., 2014, 43, 54565467.

20 H. Li, M. Eddaoudi, M. O'Keeffe and O. M. Yaghi, Nature, 1999, 402, 276-279.

21 C. K. Brozek and M. Dincă, Chem. Sci., 2012, 3, 2110.

22 C. K. Brozek and M. Dincă, J. Am. Chem. Soc., 2013, 135, 12886-12891.
23 C. K. Brozek, V. K. Michaelis, T.-C. Ong, L. Bellarosa, N. López, R. G. Griffin and M. Dincă, ACS Cent. Sci., 2015, 1, 252-260.

$24 \mathrm{~J}$. W. Herndon, Niobium(Iv) Chloride in Encyclopedia of Reagents for Organic Synthesis, John Wiley \& Sons, Ltd, 2001.

25 S. S. Kaye, A. Dailly, O. M. Yaghi and J. R. Long, J. Am. Chem. Soc., 2007, 129, 14176-14177.

26 B. Chen, X. Wang, Q. Zhang, X. Xi, J. Cai, H. Qi, S. Shi, J. Wang, D. Yuan and M. Fang, J. Mater. Chem., 2010, 20, 3758.

27 J. Hafizovic, M. Bjørgen, U. Olsbye, P. D. C. Dietzel, S. Bordiga, C. Prestipino, C. Lamberti and K. P. Lillerud, J. Am. Chem. Soc., 2007, 129, 3612-3620.

28 T. A. Dougherty, PhD thesis, Iowa State University, 1967.

29 R. E. McCarley and B. A. Torp, Inorg. Chem., 1963, 2, 540-546. 30 M. D. Korzyński, L. Braglia, E. Borfecchia, C. Lamberti and M. Dincă, Inorg. Chem., 2018, 57, 13998-14004.

31 S. Bordiga, E. Groppo, G. Agostini, J. A. van Bokhoven and C. Lamberti, Chem. Rev., 2013, 113, 1736-1850.

32 K. Manna, P. Ji, Z. Lin, F. X. Greene, A. Urban, N. C. Thacker and W. Lin, Nat. Commun., 2016, 7, 12610.

33 S. Yuan, L. Zou, H. Li, Y.-P. Chen, J. Qin, Q. Zhang, W. Lu, M. B. Hall and H.-C. Zhou, Angew. Chem., Int. Ed., 2016, 55, 10776-10780.

34 Z. Li, A. W. Peters, V. Bernales, M. A. Ortuño, N. M. Schweitzer, M. R. DeStefano, L. C. Gallington, A. E. Platero-Prats, K. W. Chapman, C. J. Cramer, L. Gagliardi, J. T. Hupp and O. K. Farha, ACS Cent. Sci., 2017, 3, 31-38.

35 M. D. Korzyński, D. F. Consoli, S. Zhang, Y. Román-Leshkov and M. Dincă, J. Am. Chem. Soc., 2018, 140, 6956-6960.

36 R. J. Holmberg, T. Burns, S. M. Greer, L. Kobera, S. A. Stoian, I. Korobkov, S. Hill, D. L. Bryce, T. K. Woo and M. Murugesu, Chem.-Eur. J., 2016, 22, 7711-7715.

37 A. Blomqvist, C. M. Araujo, P. Srepusharawoot and R. Ahuja, Proc. Natl. Acad. Sci. U. S. A., 2007, 104, 20173-20176.

38 S. S. Han and W. A. Goddard, J. Am. Chem. Soc., 2007, 129, 8422-8423.

39 S. J. Kolmann, B. Chan and M. J. T. Jordan, Chem. Phys. Lett., 2008, 467, 126-130.

40 M. Dixit, T. A. Maark and S. Pal, Int. J. Hydrogen Energy, 2011, 36, 10816-10827.

41 N. T. T. Ha, O. V. Lefedova and N. N. Ha, Russ. J. Phys. Chem. A, 2016, 90, 220-225.

42 S. Chaemchuem, Z. Kui and F. Verpoort, CrystEngComm, 2016, 18, 7614-7619.

43 M. S. Denny, L. R. Parent, J. P. Patterson, S. K. Meena, H. Pham, P. Abellan, Q. M. Ramasse, F. Paesani, N. C. Gianneschi and S. M. Cohen, J. Am. Chem. Soc., 2018, 140, 1348-1357.

44 C. Z. Andrade, Curr. Org. Synth., 2004, 1, 333-353.

45 T. Saito, H. Nishiyama, H. Tanahashi, K. Kawakita, H. Tsurugi and K. Mashima, J. Am. Chem. Soc., 2014, 136, 5161-5170.

46 O. Mathon, A. Beteva, J. Borrel, D. Bugnazet, S. Gatla, R. Hino, I. Kantor, T. Mairs, M. Munoz, S. Pasternak, F. Perrin and S. Pascarelli, J. Synchrotron Radiat., 2015, 22, 1548-1554. 